\title{
AVALIAÇÃO DAS ALTERAÇÕES PLEUROPULMONARES APÓS A INJEÇÃO DE ÓLEO DE RESINA DE COPAÍBA, EXTRATO AQUOSO DE CRAJIRU E POLIVINILPIRROLIDONA IODADO (PVPI) NA PLEURA E PARÊNQUIMA PULMONAR DE RATOS
}

\author{
EVALUATION OF THE PLEUROPULMONARY ALTERATIONS AFTER INJECTION OF \\ COPAIBA OIL, AQUEOUS EXTRACT OF CRAJIRU AND IODINE PVP IN THE PLEURAL \\ SPACE OF MICE
}

Fernando Luiz Westphal. TCBC-AM¹; Luiz Carlos de Lima²; Ricardo Alexandre Guimarães ${ }^{3}$; Risonilce Fernandes Silva de Souza ${ }^{4}$; Saulo Brasil do Couto ${ }^{5}$; Sílvia Rocha Nakajima ${ }^{5}$

\begin{abstract}
RESUMO: Objetivo: Avaliar as alterações anatomopatológicas e histopatológicas da pleura e do parênquima pulmonar após a injeção de óleo de copaíba, extrato aquoso de crajiru e polivinilpirrolidona iodado (PVPI) no espaço pleural de ratos. Método: Foram utilizados 128 Rattus norvegicus var. Wistar, machos, com peso médio 198,9g ( $\pm 24,9 \mathrm{~g})$, randomizados em quatro grupos: copaíba, PVPI, crajiru e simulação. As substâncias foram injetadas no espaço pleural direito dos animais, os quais foram mortos em 24 h, 48 h, 72 h e 504 h, para análise macro e microscópica da pleura visceral e pulmão direito. Resultados: Macroscopicamente, observou-se intensa reação pleuro-pulmonar no grupo copaíba com significância estatística $(\mathrm{p}=0,001)$ em relação aos outros grupos e entre os diferentes momentos. Microscopicamente, a espessura pleural apresentou maior aumento no grupo copaíba com significância estatística nos tempos 72 h e 504 h. O PVPI provocou reação inflamatória aguda em 24 h e 48 h com melhora em 72 h, porém, na última observação, evidenciou-se lesão crônica pulmonar. O crajiru apresentou-se pouco irritativo e sem significância em relação aos demais. Conclusão: A copaíba mostrou-se muito irritante; o PVPI, moderadamente irritante, e o extrato aquoso de crajiru apresentou pouca reação inflamatória na pleura e parênquima pulmonar dos animais de experimentação (Rev. Col. Bras. Cir. 2007; 34(3): 170-176).

Descritores: Plantas medicinais; Extratos vegetais; Fitoterapia; Pulmão; Pleura.
\end{abstract}

\section{INTRODUÇÃO}

A Amazônia é a maior reserva de produtos naturais com efeitos fitoterápicos do planeta e a sua população emprega-os empiricamente para esses fins. A copaíba e o crajiru são plantas cujos produtos são usados popularmente para inibir a inflamação e promover a cicatrização quando usados por via oral ou topicamente ${ }^{1,2}$. Contudo, há poucas descrições na literatura que comprovem seus verdadeiros efeitos terapêuticos e colaterais; além disso, não há qualquer relato de suas atividades sobre a pleura e o parênquima pulmonar quando essas substâncias são injetadas no espaço pleural.

A Copaifera multijuga, pertencente à família Leguminosae - Caesalpinoideae, conhecida como copaibeira, é árvore de grande porte e de cujo tronco é extraído um óleo-resina chamado óleo de copaíba. Esse bálsamo é utilizado popularmente como antiinflamatório, analgésico, anestésico, anti-séptico, cicatrizante e, ainda, no tratamento de infecções broncopulmonares. Em sua composição quími- ca, há uma mistura de sesquiterpenos e diterpenos em concentrações variadas ${ }^{1,3}$.

A Arrabidaea chica, da família Bignoniaceae, conhecida como crajiru, é empregada em inflamações uterinas e vaginais e como antianêmico, antiinflamatório, antihemorrágico e cicatrizante. Sua composição química contém diversas substâncias, tais como as saponinas, os quininos, as flavonas. $\mathrm{O}$ estudo da composição mineral identificou a presença de cálcio $(4,150 \mathrm{mg} \%)$, fósforo $(30 \mathrm{ug} \%)$, manganês $(0,26 \mathrm{ug} \%)$ e ferro $(12,4 \mathrm{mg} \%)^{2,4,5}$.

O polivinilpirrolidona iodado (PVPI) é um anti-séptico destinado à degermação da pele ${ }^{6} \mathrm{e}$, em baixas concentrações, pode ser útil como agente antibacteriano tópico, no tratamento de feridas cirúrgicas e úlceras cutâneas, sem inibir a atividade fibroblástica, essencial para o processo cicatricial ${ }^{7}$.

A solução de PVPI é intensamente absorvida por mucosas, o que induz a um incremento na concentração sérica de iodo, captado pela glândula tireóide, e pode ser detectado

1. Professor Adjunto do Departamento de Cirurgia da Universidade Federal do Amazonas e Universidade Estadual do Amazonas; Chefe do Serviço de Cirurgia Torácica do Hospital Universitário Getúlio Vargas; Mestre em Cirurgia Geral - Setor Tórax pela UFRJ; Doutor em Medicina pela UNIFESP.

2. Professor Adjunto da Universidade Estadual do Amazonas; Médico Assistente do Serviço de Cirurgia Torácica do HUGV; Mestre e Doutor em Medicina pela UNIFESP.

3. Médico Patologista do Departamento de Patologia da Universidade Federal do Amazonas; Mestre em Patologia Tropical pela UFAM.

4. Zootecnista do Instituto Nacional de Pesquisa do Amazônia.

5. Acadêmico de Medicina

Recebido em: 07/10/2006

Aceito para publicação em 11/12/2006

Conflito de interesses: nenhum

Fonte de financiamento: $\mathrm{CNPq}$

Trabalho realizado no Serviço de Cirurgia Torácia - HUGV e Instituto Nacional de Pesquisa da Amazônia. 
na saliva, no suor e no leite materno ${ }^{8}$. Além de sua utilização como anti-séptico, o PVPI pode ser utilizado no tratamento de processos infecciosos no mediastino pelo método de irrigação diluído em solução salina $0,9 \%$.

Este trabalho foi idealizado para verificar a possibilidade de utilização do óleo de copaíba e crajiru no tratamento das cavidades pleurais empiemáticas, considerando-se as características anti-sépticas destas substâncias ${ }^{1,2}$.

O objetivo do presente estudo é analisar as alterações anatomopatológicas e histopatológicas desencadeadas na pleura e parênquima pulmonar de ratos pelo óleo de copaíba, extrato aquoso de crajiru e PVPI.

\section{MÉTODO}

Este trabalho foi desenvolvido no Biotério Central do Instituto Nacional de Pesquisas da Amazônia - INPA e no Serviço de Patologia da Faculdade de Ciências da Saúde da Universidade Federal do Amazonas - UFAM. O experimento foi supervisionado por zootecnista do Biotério Central do INPA, a fim de garantir condições sanitárias adequadas, conforto e bem-estar aos animais, segundo o Colégio Brasileiro de Experimentação Animal.

Antes do início do experimento propriamente dito, foi desenvolvido um plano-piloto com a finalidade de avaliar sua exeqüibilidade.

Os 128 Rattus norvegicus var. Wistar, machos, com peso médio $198,9 \mathrm{~g}( \pm 24,9 \mathrm{~g})$, dos quais 43 apresentavam peso menor de $200 \mathrm{~g}$, foram distribuídos em quatro grupos contendo 32 ratos em cada: Grupo 1 (óleo de resina de copaíba); Grupo 2 (PVPI); Grupo 3 (extrato aquoso de crajiru) e Grupo 4 (simulação: solução salina a $0,9 \%$ ). Estes grupos foram subdivididos em quatro subgrupos, conforme o tempo de morte dos ratos: $24 \mathrm{~h}, 48 \mathrm{~h}, 72 \mathrm{~h}$ e $504 \mathrm{~h}$, de forma randomizada.

O cálculo da dose ideal das substâncias foi feito com base na proporção ponderal entre um homem adulto médio de $70 \mathrm{~kg}$ e o rato macho médio de $198,9 \mathrm{~g}$, sendo o produto dessa "regra de três" multiplicado por dois, devido à cavidade pleural dupla deste animal. Determinou-se que ratos com menos de $200 \mathrm{~g}$ receberiam a injeção de $0,35 \mathrm{ml}$ das substâncias e ratos com mais de $200 \mathrm{~g}$ receberiam a injeção de $0,4 \mathrm{ml}$.

Os ratos foram pesados, tricotomizados no local da incisão e, sob anestesia inalatória com éter dietílico, realizouse incisão de $5 \mathrm{~mm}$, precedida de assepsia local com álcool iodado a $2 \%$, na região subxifóide e injetadas as substâncias com agulha de anestesia peridural Tuohy $12 \mathrm{~F} \mathrm{BD}^{\circledR}$, via transdiafragmática, na cavidade pleural direita. Para uma recuperação mais rápida e um menor índice de mortalidade no pósanestésico, cada rato foi colocado numa campânula enriquecida com oxigênio, logo após a injeção, por dez minutos.

Os animais foram mortos em grupos de 32 ratos com 24 h, 48 h, 72 h e 504 h, após a injeção da substância selecionada, pela técnica de exposição ao éter, em câmara fechada, até a parada cardiorrespiratória.

A seguir, procedeu-se à incisão mediana do tórax e abdome e à observação das alterações macroscópicas, as quais foram classificadas em graus, conforme descrito por Tonietto ${ }^{11}$, de acordo com a modificação da cavidade pleural e do parênquima pulmonar:

Grau 0: nenhuma alteração macroscópica;

Grau 1: presença de exsudato, sem reação de fibrina ou aderências;

Grau 2: presença de exsudato, com reação de fibrina leve e aderências tênues;

Grau3: presença de exsudato, com reação de fibrina leve e aderências moderadas;

Grau 4: ausência de exsudato, pulmão encarcerado pela intensa reação de fibrina e inúmeras aderências.

Feita a análise macroscópica, o pulmão direito e a pleura parietal da região da parede anterior direita do tórax foram removidos para que fossem analisados microscopicamente. Foram observadas alterações na pleura, no parênquima pulmonar e na espessura pleural, classificando-as conforme descrito por Haddad ${ }^{12} \mathrm{em}$ :

Grau de reação:

Grau 0: nenhuma alteração microscópica;

Grau 1: reação inflamatória leve;

Grau 2: reação inflamatória moderada;

Grau 3: reação inflamatória intensa.

Além dessa, o patologista responsável pelo estudo microscópico classificou as alterações em reações agudas (A) ou crônicas (C).

Os resultados obtidos foram avaliados por meio da análise de dados categorizados pelo teste da razão da máxima verossimilhança, com o objetivo de avaliar a existência de associação entre as substâncias e o tipo de alteração provocada por elas, no nível de significância de $5 \%$.

Para a avaliação da variável espessura da pleura foi considerado o delineamento em parcela subdividida no tempo, na técnica não-paramétrica e em comparações múltiplas. O nível de significância também adotado foi o de $5 \%$.

\section{RESULTADOS}

\section{Análise Macroscópica}

Conforme demonstrado na tabela 1, comparando-se os diferentes grupos, quanto ao exame macroscópico no Grupo de 24 h, observa-se que a copaíba e o PVPI foram as substâncias que mais provocaram alterações, com significância estatística $(\mathrm{p}=0,001)$.

No Grupo de 48 h, o Grupo Copaíba destacou-se como o mais reativo na cavidade torácica com $50 \%$ dos ratos já apresentando encarceramento pulmonar (Grau 4) $(\mathrm{p}=0,001)$.

Durante a análise macroscópica no Grupo de 72 h, o Grupo PVPI teve cinco $(62,5 \%)$ ratos no Grau 0. O Grupo copaíba apresentou quatro ratos $(66,67 \%)$ no Grau 4 , um $(16,67 \%)$ no Grau 0, em cuja musculatura foi encontrado o óleo injetado, e outro $(16,67 \%)$ no Grau 2. $(\mathrm{p}=0,001)$. Dois componentes do Grupo Copaíba morreram logo após a anestesia.

$\mathrm{Na}$ avaliação de $504 \mathrm{~h}$, todos os inseridos no Grupo Copaíba se enquadravam no Grau 4. Um animal deste grupo não sobreviveu ao pós-operatório imediato, provavelmente por pneumotórax. 
Tabela 1 - Alterações macroscópicas em relação ao tempo do experimento.

\begin{tabular}{lccccc}
\hline & & \multicolumn{2}{c}{ ANÁLISE MACROSCÓPICA } \\
\cline { 2 - 5 } & \multicolumn{2}{c}{ Escala no tempo de 24 horas } \\
\hline Reação Inflamatória & \multicolumn{1}{c}{ Grupo } & Simulação & TOTAL \\
\cline { 2 - 5 } & PVPI & Crajiru & Copaíba & 8 & $\mathbf{1 8}$ \\
Grau 0 & 0 & 8 & 2 & 0 & $\mathbf{8}$ \\
Grau 1 & 4 & 0 & 4 & 0 & $\mathbf{6}$ \\
Grau 2 & 4 & 0 & 2 & 0 & $\mathbf{0}$ \\
Grau 3 & 0 & 0 & 0 & 0 & $\mathbf{0}$ \\
Grau 4 & 0 & 0 & 0 & $\mathbf{8}$ & $\mathbf{3 2}$
\end{tabular}

TOTAL

Escala no tempo de 48 horas

\begin{tabular}{lccccc}
\hline Reação Inflamatória & \multicolumn{5}{c}{ Grupo } \\
\cline { 2 - 6 } & PVPI & Crajiru & Copaíba & Simulação & TOTAL \\
\hline Grau 0 & 3 & 8 & 0 & 8 & $\mathbf{1 9}$ \\
Grau 1 & 3 & 0 & 0 & 0 & $\mathbf{3}$ \\
Grau 2 & 2 & 0 & 1 & 0 & $\mathbf{3}$ \\
Grau 3 & 0 & 0 & 3 & 0 & $\mathbf{3}$ \\
Grau 4 & 0 & 0 & 4 & 0 & $\mathbf{4}$ \\
TOTAL & $\mathbf{8}$ & $\mathbf{8}$ & $\mathbf{8}$ & $\mathbf{8}$ & $\mathbf{3 2}$
\end{tabular}

Escala no tempo de 72 horas

\begin{tabular}{lccccc}
\hline Reação Inflamatória & \multicolumn{5}{c}{ Grupo } \\
\cline { 2 - 6 } & PVPI & Crajiru & Copaíba & Simulação & TOTAL \\
\hline Grau 0 & 5 & 8 & 1 & 8 & $\mathbf{2 2}$ \\
Grau 1 & 3 & 0 & 0 & 0 & $\mathbf{3}$ \\
Grau 2 & 0 & 0 & 1 & 0 & $\mathbf{1}$ \\
Grau 3 & 0 & 0 & 0 & 0 & $\mathbf{0}$ \\
Grau 4 & 0 & 0 & 4 & 0 & $\mathbf{4}$ \\
TOTAL & $\mathbf{8}$ & $\mathbf{8}$ & $\mathbf{6}$ & $\mathbf{8}$ & $\mathbf{3 0}$
\end{tabular}

Escala no tempo de 504 horas

\begin{tabular}{lccccc}
\hline Reação Inflamatória & \multicolumn{5}{c}{ Grupo } \\
\cline { 2 - 6 } & PVPI & Crajiru & Copaíba & Simulação & TOTAL \\
\hline Grau 0 & 0 & 4 & 0 & 8 & $\mathbf{1 2}$ \\
Grau 1 & 3 & 1 & 0 & 0 & $\mathbf{4}$ \\
Grau 2 & 5 & 3 & 0 & 0 & $\mathbf{8}$ \\
Grau 3 & 0 & 0 & 0 & 0 & $\mathbf{0}$ \\
Grau 4 & 0 & 0 & 7 & 0 & $\mathbf{7}$ \\
TOTAL & $\mathbf{8}$ & $\mathbf{8}$ & $\mathbf{7}$ & $\mathbf{8}$ & $\mathbf{3 1}$ \\
\hline
\end{tabular}

$p<0,001$

Análise de dados categorizados pelo teste da máxima razão de verossimilhança.

Quanto ao exame macroscópico do Grupo PVPI, no mesmo momento, cinco ratos $(62,5 \%)$ encontravam-se no Grau 2 e três $(37,5 \%)$ no Grau 1.

No Grupo Crajiru, encontrou-se alteração macroscópica apenas no tempo de $504 \mathrm{~h}$, em que quatro $(50 \%)$ animais enquadravam-se no Grau 0 , um (12,5\% ) no Grau 1 e três $(37,5 \%)$ Grau 2. Notou-se também, neste tempo, que a superfície externa de alguns pulmões se encontrava toda revestida por pontos escuros, provavelmente pela impregnação por grânulos do extrato aquoso de crajiru.

O Grupo Simulação mostrou-se eficiente para tal finalidade com $100 \%$ dos ratos sem alterações patológicas em todos os tempos avaliados ( $24 \mathrm{~h}, 48 \mathrm{~h}, 72 \mathrm{~h}$ e $504 \mathrm{~h}$ ). 


\section{Análise Microscópica}

Quanto ao espessamento pleural, observou-se, ao longo do tempo, que foi mais expressivo no grupo tratado com copaíba, nos tempos de 72 h e 50 h 4, conforme demonstrado na Figura $1(\mathrm{p}<0,01)$.

O Grupo Copaíba, no tempo de 24 h, foi o que mais apresentou alterações inflamatórias moderadas, enquanto no Grupo PVPI, a maior parte dos ratos evidenciou processos reacionais leves $(\mathrm{p}=0,001)$. (Tabela 2$)$

$\mathrm{Na}$ análise microscópica no tempo 48 h, os Grupos PVPI e Crajiru tiveram o mesmo comportamento, com sete ratos sem anormalidades. No Grupo Copaíba, todos mostraram processos irritativos do tipo inflamatório e apenas um rato $(12,5 \%)$ exibiu reação do tipo crônica e intensa (Grau 3C). $(\mathrm{p}<0,001)$.

No tempo 72 h, o Grupo Crajiru mostrou-se mais reativo do que o Grupo PVPI, com dois ratos (25\%) portadores de alterações Grau $1 \mathrm{C}$, enquanto seis $(75 \%)$ componentes do último apresentavam Grau 1A. No Grupo Copaíba, quatro ratos $(57 \%)$ encontravam-se no Grau 2A.

No exame microscópico no tempo 504 h , três $(37,5 \%)$ ratos do Grupo Copaíba tiveram que ser excluídos: um, devido a erro de punção; outro, por ter sido acometido de pneumonia e o último, por obtenção de tecido inapropriado para a análise histológica. Dois ratos (40\%) encontravam-se no Grau 3C. Nesse mesmo tempo de morte, um deles inserido no Grupo PVPI e outro no Crajiru também foram excluídos, em conseqüência de pneumonia e erro na punção, respectivamente.

Observou-se no Grupo Crajiru a presença de pigmentos marrons enegrecidos na superfície da pleura e do parênquima pulmonar, correspondendo à análise macroscópica à presença de pontilhados escuros na superfície pulmonar.

\section{DISCUSSÃO}

Neste estudo, foram utilizadas duas substâncias naturais conhecidas, popular e cientificamente, por suas ações antiinflamatórias e cicatrizantes, que, apesar de amplamente empregadas com essas e outras finalidades, não foram utilizadas no espaço pleural por desconhecimento dos seus efeitos, tanto na pleura quanto no parênquima pulmonar, quando injetadas no revestimento seroso tóraco-pulmonar. Além dessas, empregou-se um anti-séptico tópico, que tem sido relatado como possível agente na indução de pleurodese.

As atividades da copaíba já foram estudadas experimentalmente. Um ensaio demonstrou que o óleo administrado por via oral reduziu o edema induzido pela carregenina na pata de ratos $^{13}$. Outros estudos experimentais demonstraram ainda seu efeito na cicatrização de suas feridas cutâneas ${ }^{14,15}$.

Com relação às alterações macro e microscópicas obtidas neste experimento, o grupo tratado com copaíba (Copaifera multijuga) desenvolveu mais reações inflamatórias em relação às outras substâncias em todas as etapas estudadas. Não encontramos relatos na literatura por nós consultada a respeito do uso dessa substância na cavidade pleural. Um estudo experimental mostrou que o produto da mesma espécie, ao ser aplicado na cavidade peritoneal, desencadeia

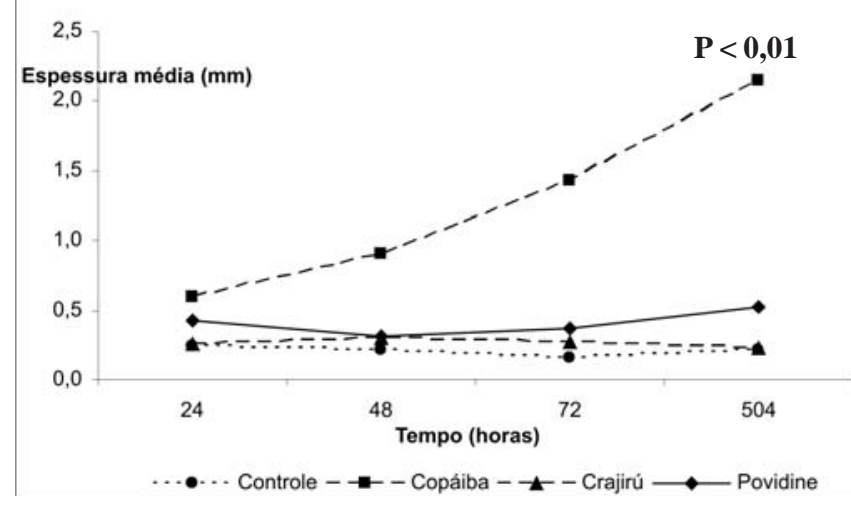

Figura 1: Espessura pleural em relação ao tempo do experimento. Delineamento em parcela subdividida no tempo, na técnica nãoparamétrica e em comparações múltiplas.

irritação, traduzida por aderências em todos os casos estuda$\operatorname{dos}^{16}$. Levando-se em consideração que a serosa abdominal assemelha-se histologicamente à pleural, presume-se que as respostas sejam similares, pois, tanto numa como noutra observou-se macroscopicamente intensa reação inflamatória, além das alterações microscópicas no parênquima pulmonar dos animais utilizados no presente estudo.

Um experimento com o crajiru em ratos albinos mostrou que doses de até $4 \mathrm{~g} / \mathrm{kg}$ de peso corporal, administradas seja por via oral e/ou intraperitoneal, não causaram letalidade. Tal achado é sugestivo de baixa toxicidade da planta².

Algumas tribos indígenas da Amazônia preparam infusão das folhas de crajiru para tratamento não só de conjuntivites, como também para lesões dérmicas secundárias, leucorréias, inflamações de útero e ovário, cólica intestinal, anemias e leucemias. Este fitoterápico possui propriedade anti-séptica obtida nos taninos, substância encontrada em abundância nesse produto ${ }^{2,4,5}$.

Não encontramos relatos na literatura do uso de crajiru na cavidade pleural. Por meio desse experimento, foi demonstrado que esse produto, quando comparado ao óleo de copaíba e ao PVPI, foi o menos reativo, comportando-se, em alguns momentos ( 24 h, 48 h e 72 h), como a solução salina do Grupo Simulação.

A outra substância estudada foi o PVPI, que demonstra ser eficaz no combate das mediastinites ${ }^{9,10}$ e na prevenção de deiscências nas anastomoses colônicas ${ }^{17}$. Além disso, é utilizado para o tratamento de peritonite, apesar dos resultados controversos ${ }^{18}$.

Há relatos ${ }^{19,20}$ do emprego de PVPI na cavidade torácica de pacientes com derrames recorrentes evidenciando que não houve necessidade de drenagem adicional desse espaço em $64,2 \%$ e $96,1 \%$ dos pacientes, respectivamente, demonstrando, assim, que esse anti-séptico é possivelmente alternativa eficaz, prontamente disponível e barata para induzir a pleurodese química ${ }^{19,20}$. Os mecanismos envolvidos na indução desse fenômeno pelo PVPI são desconhecidos e supõe-se que estão relacionados ao seu $\mathrm{pH}$ ácido ${ }^{8}$.

Os achados da ação do PVPI na cavidade pleural, nesta pesquisa, diferem da literatura, pois a maioria dos ratos apresentava, macroscopicamente, exsudato com aderências 
Tabela 2 - Alterações microscópicas em relação ao tempo do experimento.

\begin{tabular}{lccccc}
\hline & & \multicolumn{2}{c}{ ANÁLISEMICROSCÓPICA } \\
\cline { 2 - 5 } & \multicolumn{2}{c}{ Escala no tempo de 24 horas } \\
\hline Reação Inflamatória & \multicolumn{1}{c}{ Grupo } & Copaíba & Simulação & TOTAL \\
\cline { 2 - 5 } & PVPI & Crajiru & 0 & 8 & $\mathbf{1 4}$ \\
\hline Grau 0 & 0 & 6 & 2 & 0 & $\mathbf{9}$ \\
Grau 1A & 5 & 2 & 4 & 0 & $\mathbf{7}$ \\
Grau 2A & 3 & 0 & 2 & 0 & $\mathbf{2}$ \\
Grau 3A & 0 & 0 & 0 & 0 & $\mathbf{0}$ \\
Grau 1C & 0 & 0 & 0 & 0 & $\mathbf{0}$ \\
Grau 2C & 0 & 0 & 0 & $\mathbf{0}$ \\
Grau 3C & 0 & 0 & $\mathbf{8}$ & $\mathbf{8}$ & $\mathbf{3 2}$
\end{tabular}

Escala no tempo de 48 horas

\begin{tabular}{lccccc}
\hline Reação Inflamatória & \multicolumn{3}{c}{ Grupo } & TOTAL \\
\cline { 2 - 6 } & PVPI & Crajiru & Copaíba & Simulação & $\mathbf{2 2}$ \\
\hline Grau 0 & 7 & 7 & 0 & 8 & $\mathbf{3}$ \\
Grau 1A & 1 & 1 & 1 & 0 & $\mathbf{2}$ \\
Grau 2A & 0 & 0 & 2 & 0 & $\mathbf{4}$ \\
Grau 3A & 0 & 0 & 4 & 0 & $\mathbf{1 6}$ \\
Grau 1C & 2 & 4 & 2 & 8 & $\mathbf{0}$ \\
Grau 2C & 0 & 0 & 0 & 0 & $\mathbf{1}$ \\
Grau 3C & 0 & 0 & 1 & 0 & $\mathbf{3 2}$ \\
TOTAL & $\mathbf{8}$ & $\mathbf{8}$ & $\mathbf{7}$ & $\mathbf{8}$ &
\end{tabular}

Escala no tempo de 72 horas

\begin{tabular}{lccccc}
\hline Reação Inflamatória & \multicolumn{5}{c}{ Grupo } \\
\cline { 2 - 6 } & PVPI & Crajiru & Copaíba & Simulação & TOTAL \\
\hline Grau 0 & 2 & 4 & 2 & 8 & $\mathbf{1 6}$ \\
Grau 1A & 6 & 2 & 0 & 0 & $\mathbf{8}$ \\
Grau 2A & 0 & 0 & 4 & 0 & $\mathbf{4}$ \\
Grau 3A & 0 & 0 & 1 & 0 & $\mathbf{1}$ \\
Grau 1C & 0 & 2 & 0 & 0 & $\mathbf{2}$ \\
Grau 2C & 0 & 0 & 0 & 0 & $\mathbf{0}$ \\
Grau 3C & 0 & 0 & 0 & 0 & $\mathbf{0}$ \\
TOTAL & $\mathbf{8}$ & $\mathbf{8}$ & $\mathbf{7}$ & $\mathbf{8}$ & $\mathbf{3 1}$
\end{tabular}

Escala no tempo de 504 horas

\begin{tabular}{lccccc}
\hline Reação Inflamatória & \multicolumn{5}{c}{ Grupo } \\
\cline { 2 - 6 } & PVPI & Crajiru & Copaíba & Simulação & TOTAL \\
\hline Grau 0 & 0 & 4 & 1 & 8 & $\mathbf{1 3}$ \\
Grau 1A & 3 & 2 & 0 & 0 & $\mathbf{5}$ \\
Grau 2A & 0 & 0 & 0 & 0 & $\mathbf{0}$ \\
Grau 3A & 0 & 0 & 0 & 0 & $\mathbf{0}$ \\
Grau 1C & 1 & 1 & 1 & 0 & $\mathbf{3}$ \\
Grau 2C & 3 & 0 & 1 & 0 & $\mathbf{4}$ \\
Grau 3C & 0 & 0 & 2 & 0 & $\mathbf{2}$ \\
TOTAL & $\mathbf{7}$ & $\mathbf{7}$ & $\mathbf{5}$ & $\mathbf{8}$ & $\mathbf{2 7}$ \\
\hline
\end{tabular}


tênues e alterações inflamatórias agudas leves ao término do experimento.

Em conclusão, a análise macroscópica revelou que o óleo de copaíba foi muito irritante para a pleura e parênquima pulmonar de ratos, com formação de inúmeras aderências, enquanto que o PVPI foi moderadamente irritante e o extrato aquoso de crajiru apresentou pouca reação inflamatória. Quanto a análise microscópica, observou-se um espessamento pleural bastante acentuado e alterações pulmonares mais evidentes no grupo do óleo de copaíba.

\begin{abstract}
Background: Copaiba oil and crajiru are Amazon phytotherapics which have anti-inflammatory and cicatrisation properties. There are no descriptions about their effects in the pleural space. Therefore, the purpose of this study is to evaluate macro and microscopic pleuropulmonary alterations after injection of copaiba's oil, aqueous extract of crajiru and iodine PVP in the pleural space of mice. Methods: 128 Rattus norvegicus var. Wistar, male, with average weight of $198.9 \mathrm{~g}( \pm 24.9 \mathrm{~g})$, were used and assigned for four groups: copaíba, PVPI, crajiru and control. These substances were injected in the right pleural space of the animals, which were killed in 24h, 48h, 72h and 504h. They were submitted to macroscopical and microscopical analysis of the lung and right visceral pleura. Results: Macroscopic evaluation showed intense pleuropulmonary reaction in the copaiba's group with statistical significance ( $p=0.001)$ in opposite to the other groups and the different studied moments $(24 h, 48 h, 72 h$ and $504 h)$. Microscopically, the pleural thickness had greater increase in the copaiba's group with statistical significance at the moments $72 h$ and 504h. The PVPI caused an acute inflammatory reaction in the beginning of the experiment (24h and $48 h$ ) with improvement in $72 h$, however, in the last observation, chronic injury was shown The crajiru's group presented little damage and without significance in relation to the other groups. Conclusion: Copaíba revealed to be greatly irritating; the PVPI was moderately irritating and the watery extract of crajiru presented little inflammatory reaction for the pleura and lung of rats.
\end{abstract}

Key words: Plants, medicinal; Plant extracts; Phytotherapy; Lung; Pleura.

\section{REFERÊNCIAS}

1. Veiga Jr VF, Maciel MAM. Plantas medicinais: cura segura? Quim Nova. 2005; 28(3): 519-28.

2. Plantas medicinais e suas aplicações na indústria. Manaus: FIEAM, 1996. p.46-8.

3. Paiva LA, Gurgel LA, Campos AR, Silveira ER, Rao VS Attenuation of ischemia/reperfusion-induced intestinal injury by oleo-resin from Copaifera langsdorffii in rats. Life Sci. 2004; 75(16):1979-87

4. Albuquerque, JM. Plantas medicinais de uso popular. Brasília: ABEAS/MEC; 1989.

5. Zorn B, García-Piñeres AJ, Castro V, Murillo R, Mora G, Merfort I. 3-Desoxyanthocyanidins from Arrabidaea chica. Phytochemistry. 2001; 56(8):831-5.

6. Agarwal R, Aggarwal AN, Gupta D. Efficacy and safety of iodopovidone pleurodesis through tube thoracostomy. Respirol. 2006;11(1):105-8.

7. Ribeiro RC, Santos OLR, Moreira AM. Interferência do uso de soluções de polivinil-pirrolidona-iodo no processo cicatricial: estudo experimental em camundongos. Folha Méd. 1995; 111(1):61-5.

8. Olivares-Torres CA, Laniado-Laborín R, Chávez-García C et al. Iodopovidine pleurodesis for recurrent pleural effusions. Chest. 2002; 122 (2):581-3.

9. Sennes LU, Tsuji, DH, Imamura R, Sancho LM, Fusco LBl. Mediastinite como complicação dos abscessos cervicais profundos: as vias de propagação. Rev Bras Otorrinolaringol. 1996; 62(2):132-45.

10. Lima LC, Reis Filho FA, Gonçalves LA, Gomes MC, Cassete L, Rabelo RC, Bernardes RC. Mediastinite em cirurgia cardíaca: análise dos fatores de risco e avaliação do tratamento utilizando irrigação contínua de PVPI a 1\%. Rev Bras Cir Cardiovasc. 1996; 11(3):183-7.

11. Tonietto T, Pila ES, Madke GR, Lima e Silva U, Pelicetti JC, Camargo JJ, Cardoso MR, Nonning RB, Cardoso PF. Empiema pleural experimental em ratos: avaliação dos efeitos do uso intrapleural de dextran-40 na fase fibrinopurulenta. J Pneumol. 1999; 25(3):147-52.

12. Haddad R. Alterações histopatológicas pulmonares na pleurodese química - estudo experimental em ratos [dissertação]. Rio de Janeiro: Universidade Federal do Rio de Janeiro; 1995.

13. Basile AC, Sertié JA, Freitas PC, Zanini AC. Anti-inflammatory activity of oleoresin from Brazilian Copaifera. J Ethnopharmacol. 1988; 22(1):101-9.

14. Brito NMB, Simões MJ, Pessoa AF, Melo MCF. Efeitos do óleo de copaíba na cicatrização de feridas cutâneas abertas em ratos. Rev Para Méd. 1998; 12(1):28-32.

15. Costa CAS, Hebling J, Lia RCC. Estudo preliminar da compatibilidade biológica do cimento de óxido de zinco e copaíba: avaliação histológica de implantes subcutâneos em ratos. Rev Fac Odontol. 1996; 37(1):24-6.

16. Souza Júnior OG, Guimarães Neto HP, Pinto NT, Santos MT, Carvalho RA. Achados macroscópicos na cavidade peritoneal de ratos, após aplicação do óleo de copaíba. Rev Para Med. 2002; 16(1):14-8.

17. Milagres LC, Araújo ID, Barral SM, Grossi GX. Efeito do uso de povidine-iodine na cicatrização de anastomoses de cólon direito de ratos. Arq Gastroenterol. 2005; 42(2): 958 .

18. Whiteside OJ, Tytherleigh MG, Thrush S, Farouk R, Galland RB. Intra-operative peritoneal lavage--who does it and why? Ann R Coll Surg Engl. 2005; 87(4):255-8.

19. Kelly-Garcia J, Roman-Berumen JF, Ibarra-Perez C. Iodopovidone and bleomycin pleurodesis for effusions due to malignant epithelial neoplasms. Arch Med Res. 1997; 28(4):583-5.

20. Morales Gómez J, Téllez Becerra JL, Martínez Ormeños JE, Villalba Caloca J. Pleurodesis con yodopovidona en el derrame pleural neoplásico. Rev Inst Nac Enfermedades Respir. 1993; 6(2):71-4. 
Como citar este artigo:

Westphal FL, Lima LC, Guimarães RA, Silva de Souza RF, Couto SB, Nakajima SR. Estudo experimental dos efeitos do óleo de resina de copaíba, extrato aquoso de crajiru e polivinilpirrolidona iodado (pvpi) na pleura e parênquima pulmonar de ratos. Rev Col Bras Cir [periódico na Internet]. 2007;34(3). Disponível em URL: http:// www.scielo.br/rcbc
Endereço para correspondência:

Universidade Federal do Amazonas

Hospital Universitário Getúlio Vargas, Serviço de Cirurgia Torácica.

Rua Apurinã, 4 - Praça 14

69010-130 - Manaus - AM

Telefone: (92) 621-6500 (ramal 6504); 9122-3356

E-mail do autor principal: f.l.westphal@uol.com.br 